\title{
A note on the effect of ultrasonic waves on droplets rising in Newtonian and non-Newtonian fluids
}

\begin{abstract}
An experimental study of Newtonian and non-Newtonian droplets rising in Newtonian and non-Newtonian fluids is presented when the droplets are subjected to external ultrasonic waves. Our results show that significant changes in the drop drag coefficient are observed when the external fluid is non-Newtonian. When the external fluid is Newtonian, for both Newtonian and non Newtonian droplets the changes in terminal speed and drag coefficient are small. Our results indicate that the ultrasound has a significant effect on the properties of non Newtonian media, probably inducing changes in the effective viscosity and in the entanglement of the suspended polymer molecules.
\end{abstract}

Keywords: viscoelastic drop, ultrasound, shear-thinning fluid, terminal velocity
Volume 2 Issue 4 - 2018

\author{
A J Mendoza Fuentes,' Rebeca Cedeño \\ Madera, ${ }^{2}$ L Moreno, ${ }^{3}$ Roberto Zenit \\ Camacho, ${ }^{3}$ Baltasar Mena Iniesta, ${ }^{4}$ Octavio \\ Manero Brito ${ }^{3}$ \\ 'Facultad de Química, Universidad Nacional Autónoma de \\ México, México \\ ${ }^{2}$ Facultad de Ingeniería, Universidad Nacional Autónoma de \\ México, México \\ ${ }^{3}$ Instituto de Investigaciones en Materiales, Universidad Nacional \\ Autónoma de México, México \\ ${ }^{4}$ Instituto de Ingenieria, Universidad Nacional Autónoma de \\ México, México
}

\begin{abstract}
Correspondence: Arturo de Jesus Mendoza Fuentes, Universidad Nacional Autónoma de México, Av. De los Insurgentes sur 3000, Circuito Interno Ciudad de México, C.P. 045 I0,Tel 52 (55) 40192487, Email ajesusmf@yahoo.com.mx
\end{abstract}

Received: August 18, 2018 | Published:September 12, 2018

\section{Introduction}

Processes such as coating, painting, blending of molten polymers, food processing and fabrication of immiscible liquid-liquid suspensions are examples in which the dynamics of droplet motion is of paramount importance in order to obtain an optimum design. ${ }^{1-3}$ Previous studies show that the dynamics of non-buoyant Newtonian drops in Newtonian fluids moving under creeping flow conditions is determined by three dimensionless parameters: the capillary number, the viscosity ratio and the density ratio. Drop deformation increases with increasing capillary number until the drop breaks up at a certain critical capillary number. This break-up occurs in the flow direction as the volume of the drop increases and the free surface of the drop deforms from a spherical shape to elongated slender forms. ${ }^{4-5}$

However, for systems involving viscoelastic components, the study of drop dynamics is not quite simple. It has been shown that in simple shear flow, viscoelastic properties of the droplet tend to reduce deformation, while in the continuous phase the deformation is reduced or increased depending on whether the Deborah number is low or high. ${ }^{6-9}$ In extensional flow, viscoelasticity of the droplet reduces deformation while its presence in the continuous surrounding phase enhances it. ${ }^{10-12}$ More recently, Zhou et al., ${ }^{13}$ and Yue et al., ${ }^{14}$ showed using finite element methods that the scheme mentioned above applies only when both capillary and viscosity ratio numbers are small; if they are large, however, the opposite effect is produced. Moreover, Sibillo et al. ${ }^{15}$ reported that the onset of drop deformation has a time dependent rheology. Finally, the critical capillary number for nonNewtonian droplet break-up increases with drop viscoelasticity and decreases with matrix viscoelasticity; the latter enhances droplet orientation and break up in the flow direction, whilst break up along the vorticity direction can be achieved at high shear rates. ${ }^{16,17}$ In addition, the droplet undergoes several cycles of oscillations before breaking up in the vorticity direction. ${ }^{18,19}$
All experimental studies mentioned above have also been predicted theoretically by different authors. ${ }^{20-25}$

However; in order to enhance mechanical properties in polymer blending, plastic recycling, and food processing, acoustic energy is used in the form of piezoelectric ultrasound in single screw and twinscrew extrusion ${ }^{26,27}$ and also for its potential use as a preservation tool in food industry. ${ }^{28}$ The propagation of small amplitude acoustic waves in fluids that contain particles is an old and much studied subject, both from the fundamental and applied points of view. Further, in some instances, theoretical formulations exist that have not received experimental corroboration. Such is the case of models where attenuation can be well predicted but fails to describe dispersion. ${ }^{29}$ In other cases, substantial differences has been observed between attenuation coefficients from those obtained experimentally. To a complete revision of literature about different aspects of theoretical

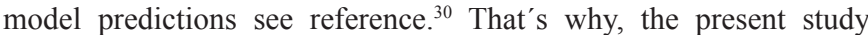
represents a good challenge to predict attenuation coefficients in these systems.

Moreover the correct prediction of attenuation and dispersion of waves can help well-established methods for detection of corrosion defects in pipelines ${ }^{31}$ and curved pipes. ${ }^{32,33}$ Additionally, compression waves can also propagate in solids. However, in solids unlike in fluids, a shearing strain produced at some point can be transmitted to adjacent layers by the strong binding between particles. This mechanism generates transverse waves also called bulk shear waves. Such is the case of drilling fluids. Very recently Ambar Veg et al., ${ }^{34,35}$ have shown nanofluids can resolve a number of issues including borehole instability and lost circulation in drilling wells. Knowing the process of attenuation and dispersion of ultrasound waves can help to obtain a better dispersion of nano-particles in the WBM.

In what follows, the dynamics of Newtonian and non-Newtonian drops rising in Glycerol (Newtonian) and Polyethylene oxide 
(Non-Newtonian) under the influence of an energy acoustic field is investigated. Physical mechanisms involved in the rising velocity of droplets are examined using rheological measurements together with simultaneous application of ultrasonic pulses.

To our knowledge, there is no previous study involving the use of ultrasonic field in droplets rising in different fluids.

\section{Experimental arrangement and test fluids}

The experimental arrangement is shown schematically in Figure 1. The device consists of a cylindrical container of $60 \mathrm{~cm}$ in length and $5 \mathrm{~cm}$ in diameter which has a test section where the drops are injected. The container has a valve at the bottom connected to a syringe which releases the droplets into the solution at the center of the container to reduce wall effects. The cylindrical container is located inside a rectangular tank made of glass, with $150 \mathrm{~cm}$ height and $20.5 \mathrm{~cm}$ width filled with water. The similarity of the refraction indices of glass and water reduces refraction effects of the curved cylinder-water interface. A piezoelectric transducer $(40 \mathrm{~Hz}$ and $50 \mathrm{Watts})$ is located at the top of the internal cylinder and its surface is in contact with that of the fluid. Measurements of rising drop velocity and shape aspect ratio were obtained using a Canon camera EOS 60D with a macro lens of $100 \mathrm{~mm}$ size. Photographs of the drops were taken during their passage through the column. The camera was mounted on one side of the viewing section and was focused on a standard glass sphere held at the center of the column. Two electric lamps (200W) were placed $30 \mathrm{~cm}$ from the backside of the column to illuminate the drop from the side. After taking photographs an image processing analysis was carried out using the image processing tools of Matlab. ${ }^{36-39}$ Shape of the drops was represented in terms of eccentricity (E) of the drop defined as the ratio of its largest horizontal dimension to the larger vertical dimension.

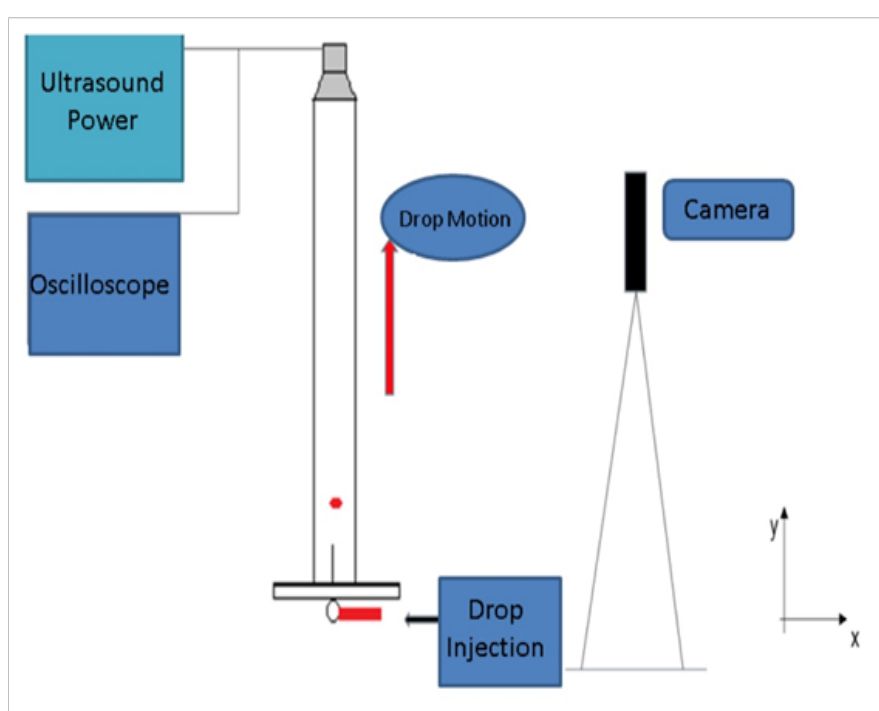

Figure I Experimental device.

Newtonian liquids: Glycerol, Glycerol-water, were used as Newtonian external fluid whilst Roshframs motor oil was used as a Newtonian droplet.

Non-Newtonian liquids: Aqueous solutions of Polyethylene oxide in distilled water were used as the external fluid and a well known and widely used solution of Polyisobutelene, in Decalin and Polybutene oil was used as the viscoelastic droplet.
Solutions of Glycerol in water at $85 \%$, Polyethylene oxide in water at $0.5 \%$ per weight, Polyisobutylene (PIB) at $2.5 \%$ in $47.5 \%$ Decalin and $50 \%$ Polybutene oil were used. The Polyisobutylene solution also known as S1 fluid was prepared following the methodology reported elsewhere in ${ }^{40}$ and is not described here. A TA Instruments G2 stresscontrolled rheometer was used to measure the viscosity and first normal stress difference flow curves, in the shear rate range of $0.01-$ $100 \mathrm{~s}-1$. Small amplitude oscillatory shear flow was used to obtain the variation of the loss G' and storage G" modulus with frequency at $22^{\circ} \mathrm{C}$. Tests were performed with the cone and plate fixture, with 4 $\mathrm{cm}$ diameter and $1.59 .14^{\circ}$ cone angle. A controlled strain rheometer (AR-1000 TA Instruments) was modified with a $40 \mathrm{~mm}$ lower plate transducer $(40 \mathrm{~Hz}$ and $75 \mathrm{~W})$. Measurements of viscosity were performed with a simultaneous application of ultrasonic pulses. The rheological behavior was determined at a constant shear rate $\left(3 \mathrm{~s}^{-1}\right)$ for a period of thirty minutes. For the first 5 minutes the fluid was allowed to reach a constant viscosity; an ultrasonic pulse was applied for a further 5 minutes, to finally stop the pulse in order to analyze its effect on the structure of the fluid. For the Newtonian fluids, the viscosities, both glycerol and motor oil showed a constant value of 0.109 and $0.479 \mathrm{~Pa}^{*} \mathrm{~s}$ respectively.

In Figures 2, the shear viscosity and first normal stress difference under simple shear are plotted vs. shear rate for polyethylene oxide (POE) and S1 fluid. At low shear rates the first Newtonian region, where the viscosity is constant, spans over one decade for POE fluid and for two decades of shear rates for S1 fluid. For moderate shear rates to high shear rates, 1 to $100 \mathrm{~s}^{-1}$, shear thinning is apparent in the POE fluid; however, shear-thinning for S1 fluid appears at high shear rates, from 10 to $100 \mathrm{~s}^{-1}$. First normal stress difference appears at shear rates greater than ten for S1 fluid. Polyethylene oxide does not show normal stress difference for the concentration used.

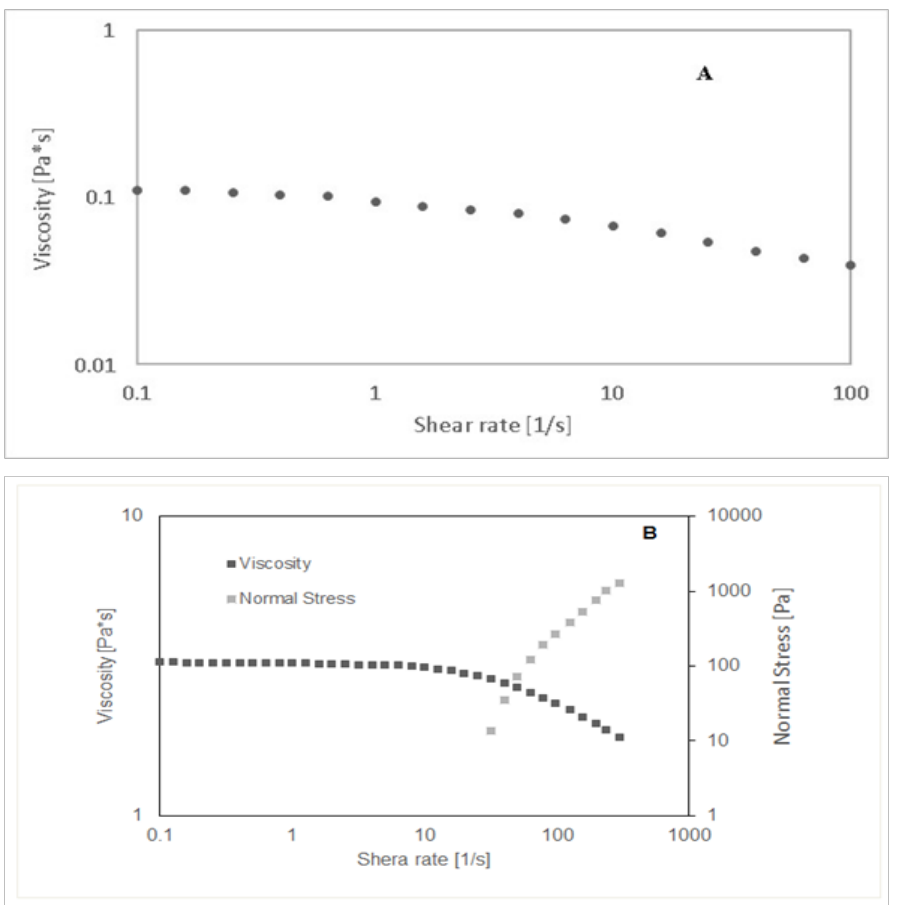

Figure 2 Shear viscosities for A) Polyethylene oxide fluid and B) SI fluid.

Figure 3 shows the linear viscoelastic data in the frequency range from 0.1 to $100 \mathrm{rad} / \mathrm{s}$ for the non-Newtonian fluids. For both fluids, 
the magnitude of loss modulus is larger than the storage modulus, implying that both fluids exhibit viscous behavior for all frequency intervals. The cross-over frequency, not seen in experimental results, implies a small value of relaxation time with concentration.
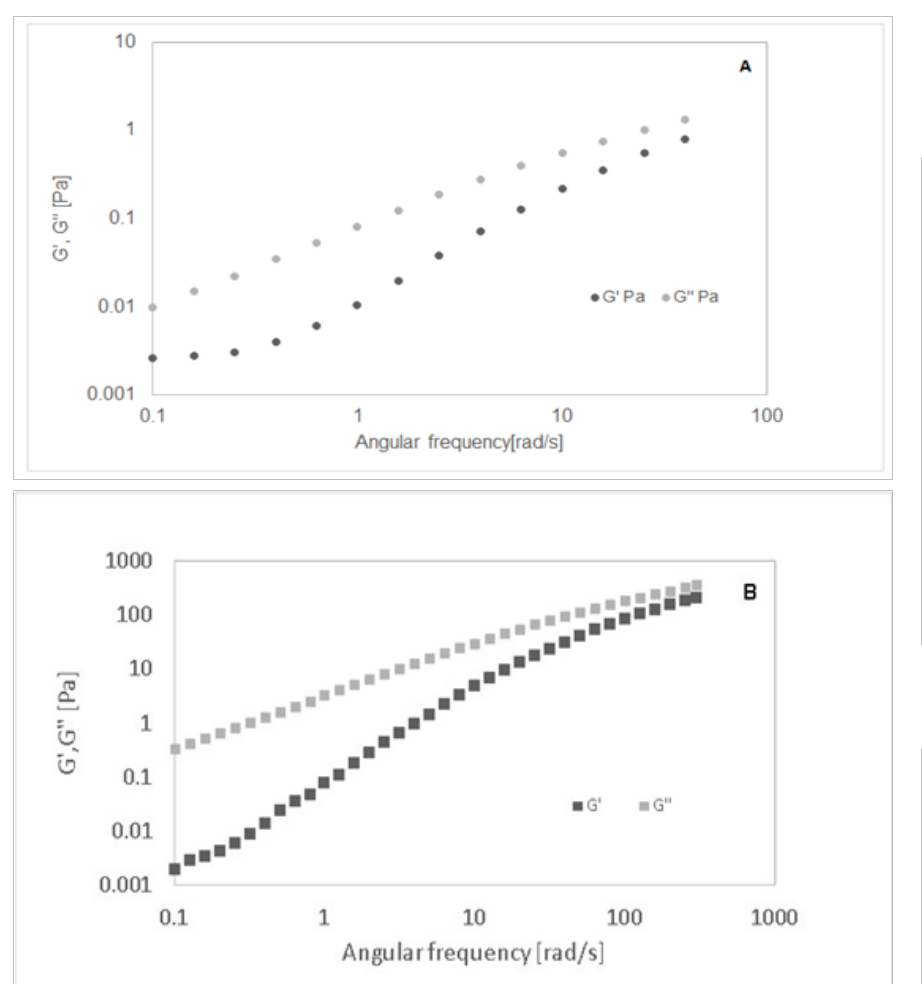

Figure 3 Loss and storage modulus for A) Polyethylene oxide fluid and B) SI fluid

\section{Results}

\section{Drop velocity as a function of time}

Experiments were performed releasing drops of a single size of glycerol and motor oil in a Newtonian fluid and in a non-Newtonian fluid. This was repeated four to six times and then the average value was used for further calculations. The characteristic velocity profile with and without the influence of power ultrasound is depicted in Figures 5 to 8 for the four systems explored.

\section{Newtonian droplet in a Newtonian external fluid}

In the case of a motor oil drop rising in glycerol (Figure 4), the velocity increases when the ultrasound field is applied. As time progresses, a slight increase in velocity may be observed; due to the approach of the droplet to the top of the container where the ultrasound field is applied and possible acoustic "cavitation" may be present. In the absence of ultrasound, the velocity is nearly constant.

\section{Newtonian droplet in a non-Newtonian external fluid}

In Figure 5, the velocity profile of the Newtonian oil drop moving along the tube shows an increment of velocity of nearly twice the original velocity when the ultrasound is applied. This increase in velocity is probably caused by displacement of physical entanglements of polyethylene oxide molecules. This causes the drop to move across a solvent which now has a lower viscosity. As the drop moves along the container we observe a decrease in velocity in the region of increasing acoustic wave presence. Again, as the drop approaches the final stage, there are less polyethylene oxide molecules due to the proximity of the piezoelectric ultrasound source. This causes the droplet to be exposed basically to the viscosity of the solvent (water), resulting in a substantial increase in its velocity. As a comparison, the velocity without the ultrasound is practically constant throughout the entire path of the droplet.

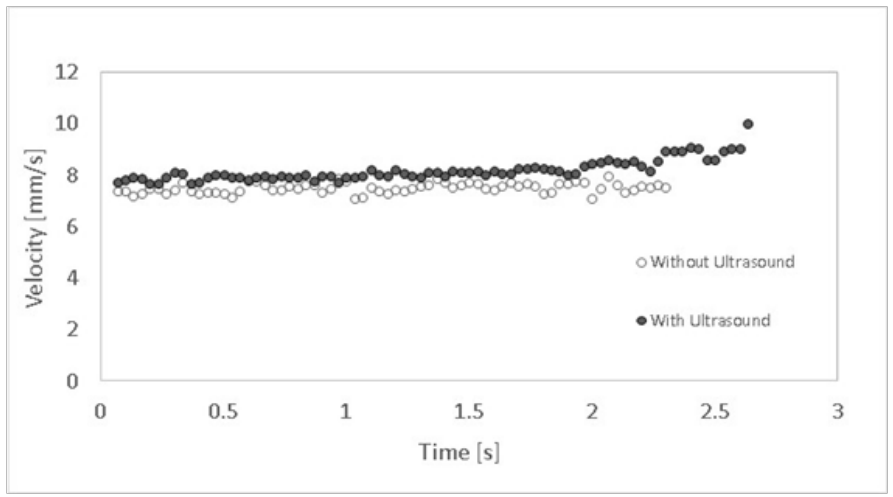

Figure 4 Drop velocity as a function of time for a motor oil drop rising in glycerol with and without the influence of an external ultrasound field.

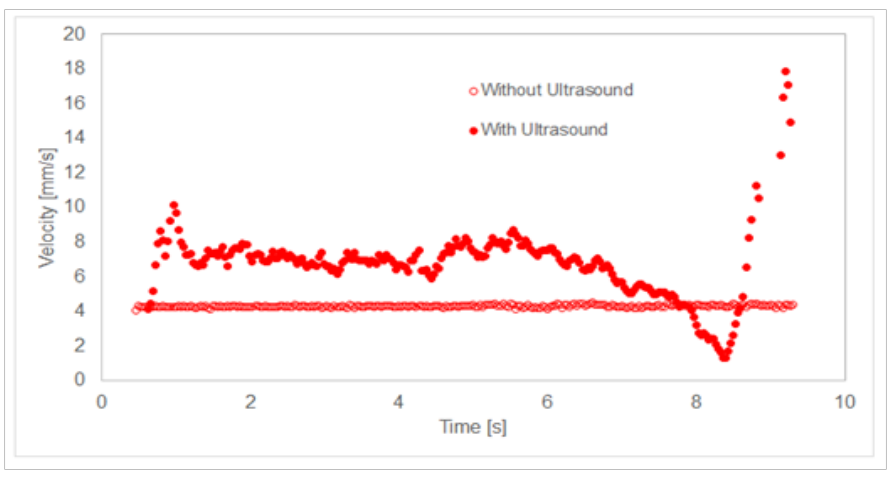

Figure 5 Drop velocity as a function of time for a motor oil drop rising in Polyethylene oxide solution with and without the influence of an external ultrasound field.

\section{Non-Newtonian droplet in a Newtonian external fluid}

In Figure 6, the velocity profile of an S1 droplet rising in glycerol is shown. In this case the droplet velocity is not significantly affected by the presence of the ultrasound field. In this case, only a small volume of non Newtonian fluid is present. Hence, the effect of ultrasound, which allegedly affects only the non Newtonian media more significantly, is minor.

\section{Non-Newtonian droplet moving in a non-Newtonian external fluid}

The last system shows an S1 droplet rising in a non-Newtonian POE fluid (Figure 7). In this case we observe a monotonic increase in droplet velocity when compared to the case where no power ultrasound is present. In this instance no reduction of drop velocity is observed, which means that all energy coming from the ultrasound waves is being used to break up physical entanglements of the POE solution. 


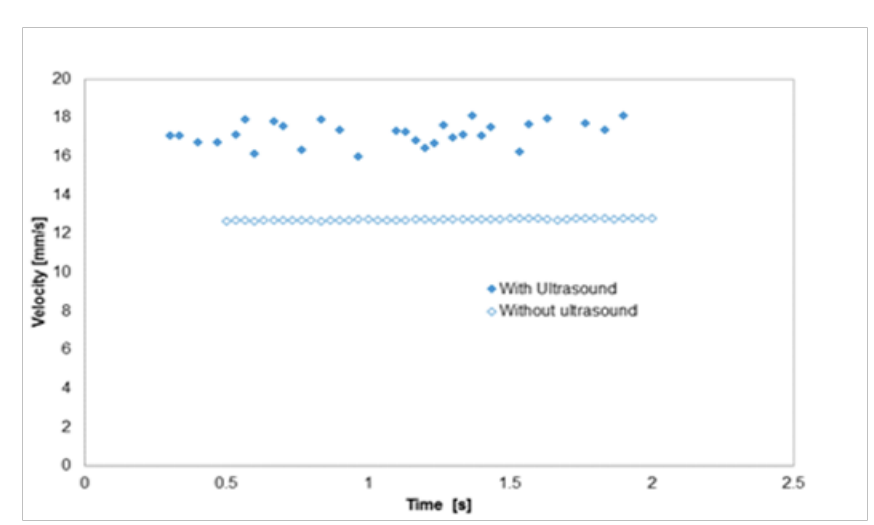

Figure 6 Drop velocity as a function of time for a SI drop rising in glycerol solution with and without the influence of an external ultrasound field.

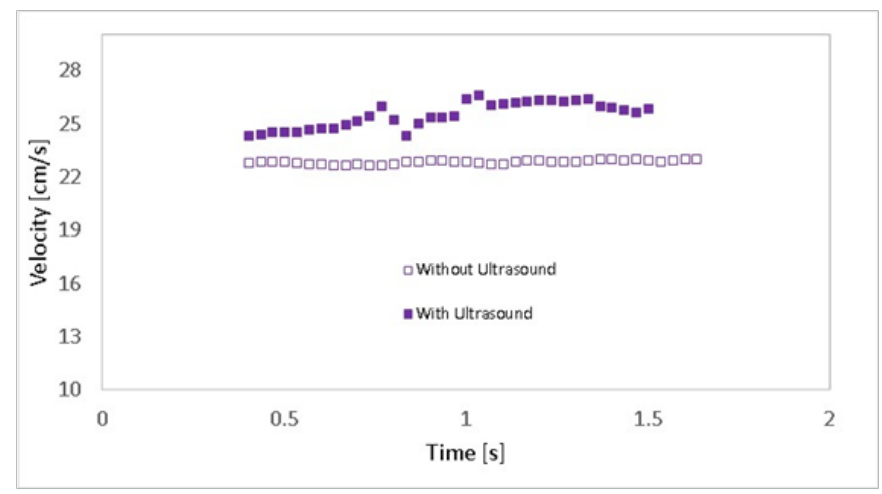

Figure 7 Drop velocity as a function of time for a polyethylene drop (SI fluid) rising in polyethylene oxide solution with and without the influence of an external ultrasound field.
It is important to note that for all cases, the droplets were not significantly deformed under the action of the ultrasound field. We measured a maximum change of $5 \%$ of the drop aspect ratio for all cases. Therefore, changes in the terminal velocity cannot be attributed to changes in shape.

\section{Dimensionless numbers}

Using the observed terminal velocity, rheological parameters, interfacial tension and physical properties of the dispersed and continuous phase three dimensionless groups, $\mathrm{Re}, \mathrm{Ca}$ and Eö were calculated. The physical properties of the droplets and the range of the experimental parameters attained for each system are shown in Table 1.

For this problem, the relevant dimensionless numbers are defined as: Reynolds number Re, Capillary number Ca, Eötvos number Eo, Viscosity ratio $\mu$ and Density ratio $\delta$ defined as: $R e=a U \rho / \eta(\dot{\gamma})$, $C a=\eta a / \Gamma, \quad E o=g a^{2} \Delta \rho / \Gamma, \quad \mu=\eta_{d} / \eta_{f}, \delta=\rho_{d} / \rho_{f}$, where $\rho_{d}$ is the drop density, $\rho_{f}$ is the fluid density, $a$ is the sphere radius, $U$ is the terminal velocity, $\eta(\dot{\gamma})$ is the shear viscosity, $\eta_{d}$ is the interfacial tension, $\eta_{d}$ drop viscosity, $\eta_{f}$ fluid viscosity.

The characteristic shear rate of the flow is: $\eta(\dot{\gamma})=U / a$. The interfacial tension was obtained measuring the surface tension of all fluids and then applying Antonoff's rule.

It should be noted that for all systems employed, interfacial forces prevail over viscous forces. This, in addition to a small value of the Eötvos number, may explain why there is no change in droplet shape during the ascent inside the different fluids considered, as predicted by Clift et al. ${ }^{41}$ Additionally, the influence of inertial forces is not significant since the Reynolds number is less than one.

Table I Physical parameters and values of dimensionless numbers for the four systems used. The dimensionless groups are calculated with the effect of ultrasound $(\mathrm{U})$ and without it $(\mathrm{WU})$

\begin{tabular}{|c|c|c|c|c|c|c|c|c|}
\hline $\begin{array}{l}\text { Drop-Fluid } \\
\text { (disperse- }\end{array}$ & $\begin{array}{l}\text { Viscosity } \\
\text { ratio }\end{array}$ & $\begin{array}{l}\text { Density } \\
\text { ratio }\end{array}$ & $\mathrm{Ca}$ & & $\mathbf{R e}$ & & Eö & \\
\hline & & & Wu & $\mathbf{U}$ & Wu & $\mathbf{U}$ & Wu & $\mathbf{U}$ \\
\hline Oil-Glycerol & 4.39 & 0.73 & $2.4 \times 10^{-5}$ & $2.8 \times 10^{-5}$ & $1.4 \times 10^{-1}$ & $2.0 \times 10^{-1}$ & $4.5 \times 10^{-4}$ & $4.9 \times 10^{-4}$ \\
\hline Oil-POE & 2.95 & 0.89 & $7.6 \times 10^{-5}$ & $7.6 \times 10^{-5}$ & $8.5 \times 10^{-1}$ & 1.01 & $5.8 \times 10^{-4}$ & $5.8 \times 10^{-4}$ \\
\hline SI-Glycerol & $\begin{array}{l}\text { Shown in } \\
\text { Figure } 6\end{array}$ & 0.72 & $2.9 \times 10^{-5}$ & $2.8 \times 10^{-5}$ & $2.1 \times 10^{-1}$ & 0.197 & $5.6 \times 10^{-4}$ & $4.9 \times 10^{-4}$ \\
\hline SI-POE & $\begin{array}{l}\text { Shown in } \\
\text { Figure } 6\end{array}$ & 0.88 & $4.4 \times 10^{-5}$ & $5.9 \times 10^{-5}$ & $5.1 \times 10^{-1}$ & 0.602 & $1.4 \times 10^{-3}$ & $1.2 \times 10^{3}$ \\
\hline
\end{tabular}

\section{Conclusions}

The experimental results described here show that there is an increase in the velocity magnitude of droplets rising in both Newtonian and non-Newtonian fluids when they are under the influence of an ultrasonic field. The velocity of the droplet varies according to the rheological nature of the fluids involved. Newtonian droplets show a virtually stationary velocity profile under ultrasound influence. NonNewtonian droplets are more susceptible to absorb ultrasound waves causing substantial changes in velocity. In the case of polyethylene oxide as an external fluid, the ultrasound waves appear to modify the internal structure, changing the rheological properties of the media.
Additional physical mechanisms that might be responsible for these variations in velocity profiles were dismissed due to the low values of Reynolds, Eötvös and Capillary numbers obtained.

\section{Acknowledgements}

R. Cedeño-Madera, acknowledges CONACYT for support during her graduate studies.

\section{Conflict of interest}

Author declares there is no conflict of interest in publishing the article. 


\section{References}

1. Tadros TF. Rheology of dispersions, principles and applications. Germany: Wiley; 2010. 216p.

2. Tadros TF, Emulsion science and technology. Germany: Wiley; 2009. $326 \mathrm{p}$.

3. Tadros TF. Colloid stability and application in pharmacy. Colloids and interface science series. Vol. 3. Germany: Wiley; 2007. 456p.

4. Rallison JM. The deformation of small viscous drops and bubbles in shear flows. Ann Rev Fluid Mech. 1984;16:45-66.

5. Stone HA. Dynamics of drop deformation and breakup in viscous fluids. Ann Rev Fluid Mech. 1994;26:65-102.

6. Mukherjee S, Sarkar K. Effects of viscosity ratio on deformation of a viscoelastic drop in a Newtonian matrix under steady shear. $J$ Non Newtonian Fluid Mech. 2009;160(2-3):104-112.

7. Li H, Sundararaj U. Experimental investigation of viscoelastic drop deformation in Newtonian matrix at high capillary number under simple shear flow. J Non Newtonian Fluid Mech. 2010;165(19-20):1219-1227.

8. Verhulst K, Moldenaers P. Drop shape dynamics of a Newtonian drop in a non-Newtonian matrix during transient and steady shear flow. $J$ Rheol. 2007;51:261-273.

9. Mukherjee S, Sarkar K. Viscoelastic drop falling through a viscous medium. Phys Fluids. 2011;23:013101.

10. Mighri F, Ajji A, Carreau PJ. Influence of elastic properties on drop deformation in elongational flow. J Rheol. 1997;41:1183-1201.

11. Milliken WJ, Leal LG. Deformation and breakup of viscoelastic drops in planar extensional flows. J Non Newtonian Fluid Mech. 1991;40(3):355379.

12. Ramaswamy S, Leal LG. The deformation of a Newtonian drop in the uniaxial extensional flow of a viscoelastic liquid. J Non-Newtonian Fluid Mech. 1999;88(1-2);149-172.

13. Zhou D, Yue P, Feng JJ. Viscoelastic effects on drop deformation in a converging pipe flow. $J$ Rheol. 2008;52:469-487.

14. Yue P, Feng JJ. Transient drop deformation upon startup of shear in viscoelastic fluids. Phys Fluids. 2005;17:123101.

15. Sibillo V, Simeone M, Guido S. Break-up of a Newtonian drop in a viscoelastic matrix under simple shear flow. Rheol Acta. 2004;43(5):449 456.

16. Li H, Sundararaja U. Does drop size affect the mechanism of viscoelastic drop breakup? Phys Fluids. 2008;20:053101.

17. Cherdhirankorn T, Lerdwijitjarud W, Siriva A, et al. Dynamics of vorticity stretching and breakup of isolated viscoelastic droplets in an immiscible viscoelastic matrix. Rheol Acta. 2004;43(3):246-256.

18. Renardy Y. Drop oscillations under simple shear in a highly viscoelastic matrix. Rheol Acta. 2008;47(1):89-96.

19. Aminzadeh M, Maleki A, Firoozabadi B, et al. On the motion of Newtonian and non-Newtonian liquid drops. Scientia Iranica $B$. 2012;19(5):1265-1278.

20. Favelukisa M, Nirb A. Non-Newtonian slender drops in a simple shear flow. J Non-Newtonian Fluid Mech. 2016;228:38-45.

21. Verhulst K, Cardinaels R, Moldenaers P, et al. Influence of viscoelasticity on drop deformation and orientation in shear flow Part 1. Stationary states. J Non-Newtonian Fluid Mech. 2009;156:29-43.

22. Favelukisa M, Lavrentevab OM, Nirb A. Deformation and breakup of a non-Newtonian slender drop in an extensional flow. $J$ Non-Newtonian Fluid Mech. 2005;125(1):49-59.
23. Ohta M, Iwasaki E, Obata E. A numerical study of the motion of a spherical drop rising in shear-thinning fluid systems. J Non-Newtonian Fluid Mech. 2003;116(1):95-111.

24. Khismatullin DB, Renardy Y. Inertia-induced breakup of highly viscous drops subjected to simple shear. Phys Fluids. 2003;1351.

25. Yue P, Feng J, Liu C, et al. Viscoelastic effects on drop deformation in steady shear. J Fluid Mech. 2005;540:427-437.

26. Sanchez Olivares G, Sanchez Solis A, Calderas F, et al. Extrusion with ultrasound applied on intumescent flame-retardant polypropylene. Pol Eng Science. 2013;53(9):2018-2026.

27. Sanchez Olivares G, Sanchez Solis A, Calderas F, et al. Flame retardant high density polyethylene optimized by on-line ultrasound extrusion. Pol Deg Stab. 2013;98(11):2153-2160.

28. Kentish S, Feng H. Applications of Power Ultrasound in Food Processing. Rev Food Sci Technol. 2014;5263-84.

29. Epstein PS, Carhart RR. The absorption of sound in suspensions and emulsions. I. Water fog in air. J Acoust Soc Am. 1953;25(3):553-565.

30. Temkin S. Attenuation and dispersion of sound in dilute suspensions of spherical particles. J Acoust Soc Am. 2000;108:126.

31. Leinov E, Lowe JS, Cawley P. Investigation of guided wave propagation and attenuation in pipe buried in sand. Journal of Soundand Vibration. 2015;347(7):96-114.

32. Norouzi M, Davoodi M, Anwar Bég O, et al. Theoretical study of Oldroyd-B visco-elastic fluid flow through curved pipes with slip effects in polymer flow processing. International Journal of Applied and Computational Mathematics. 2018;4:108.

33. Nasir Ali , Zaman A, Sajid M, et al. Numerical Simulation of timedependent non-Newtonian nano-pharmacodynamic transport phenomena in a tapered overlapping stenosed artery. Nano Sci Technol Int J. 2018;9(3):247-282.

34. Anwar Bég O, Sanchez Espinoza DE, Kadir A, et al. Experimental study of improved rheology and lubricity of drilling fluids enhanced with nano-particles. Applied Nanoscience. 2018;8(5):1069-1090.

35. Umamaheswar M, Varma SVK, Raju MC. Unsteady MHD Free Convective Visco-Elastic Fluid Flow Bounded by an Infinite Inclined Porous Plate in the Presence of Heat Source, Viscous Dissipation and Ohmic Heating. International Journal of Advanced Science and Technology. 2013;61:39-52.

36. Wanchoo RK, Sharma SK, Gupta R. Shape of a Newtonian liquid drop moving through an immiscible quiescent non-Newtonian liquid. Chem Eng Proc. 2003;42(5):387-393.

37. Hugli H, Gonzalez J. Drop volume measurement by vision. SPIE Proceedings; USA: Machine Vision Applications in Industrial Inspection VIII. 2000;3966:60-66.

38. Volkov PK. Hydrodynamics of rising bubbles and drops. J Eng Phys Thermo. 1994;66(1):85-113.

39. Ninomiya N, Yasuda K. Visualization and PIV Measurement of the Flow around and inside of a Falling Droplet. J Visual. 2006;9(3):257-264.

40. Liang RF, Mackley MR. Rheological characterization of the time and strain dependence for polyisobutylene solutions. J Non-Newtonian Fluid Mech. 1994;52(3):387-405.

41. Clift R, Grace JR, Weber ME. Bubbles, Drops, and Particles. New York: Academic Press; 1978. 380p. 